\title{
Flow immunophenotyping features of crisis phase of chronic myeloid leukemia in childhood: do we really care?
}

\author{
* Namrata N Rajkumar', *Raghvendra H Vijay ${ }^{1}$, Vijay C Raghu², Lakshmiah K C \\ ${ }^{1}$ Dr Namrata N Rajkumar, Assistant Professor of Pathology, ${ }^{1}$ Dr Raghavendra H .V. Associate Professor of Pathology, \\ ${ }^{2}$ Vijay C. Raghu, Assistant Professor, Department Biostatistics, ${ }^{3}$ Dr Lakshmiah K Chinnagiriyappa. Prof \& head \\ Department of Medical Oncology. All are affiliated with Kidwai Memorial Institute of Oncology Bangalore, Karnataka, \\ India
}

*Dr Raghavendra H.V. and *Dr Namrata N. R. have contributed equally for study.

Address for Correspondence: Dr. Namrata N R, Assistant Professor, Pathology, KMIO, Bangalore, Karnataka, India. Email:nrnamrata@yahoo.com

\begin{abstract}
Objective: Chronic leukemias are rare in childhood \& CML is extremely rare in children. Imunophenotypic studies have a limited role in the diagnosis of CML but are increasingly being used in CML blast transformation. Purpose of the study was determine the clinical and laboratory and Flow immunophenotyping (FIC) features with Mutational analysis of blast transformation of CML in children. Methods: 11 years analysis was done 187 cases of suspected CML were studied in children and adolescents. Patients were evaluated at KMIO between 2004 to 2015. 97 cases had Bone marrow diagnosis of CML. 22 cases peripheral smear was suggestive of blastic phase CML $(20 \%)$ were chosen for the study. Bone marrow confirmation was available in all the cases. Cytogenetics and Molecular confirmation was also available in all cases. FIC was done in 8/22(36\%) cases. Mutations were studied in 7 cases. Results: The disease predominantly affected older children more than 10 years 16/22(72\%). Male sex predilection was seen. Gender ratio was 1.4: 1. Most predominant clinical sign was splenomegaly. Leucocyte count $>100 \mathrm{X} 10^{9} / \mathrm{L}$ was seen in all cases. Peripherals smear suggested CML in all 22cases and bone marrow aspiration confirmed the diagnosis.17 Cases were at diagnosis. 5 Cases progressed to blastic phase from chronic phase. Median year of transformation was 4 years. In 22 cases Phildelphia chromosome was noted and 5 cases revealed additional markers PCR revealed p210 transcript in all cases. In 8 cases in the blastic phase Flow cytometry immunophenotype was done. 5 cases were myeloid blastic phase, single case was mixed phenotype, 2 cases were lymphoid blastic phase. Conclusion: Imatinib highly effective in children with advanced phase of CML. This is the largest, exclusive first reported series of blastic phase of CML in children from a single center. Only 5 cases received Imatinib, All 5cases attained remission; Cases are on follow up and continue to be in remission after a mean of 6 months.
\end{abstract}

Key words: CML: chronic myeloid leukemia, childhood, polymerase chain reaction. FCI: Flowcytometry immunophenotyping, Blastic phase

\section{Introduction}

Chronic Myeloid Leukemia (CML) is a stem cell disorder and is clonal. Balanced translocation between the long arm of chromosome [9] and $22, \mathrm{t}(9: 22)(\mathrm{q} 34 ; \mathrm{q} 11)$ also called the Phildelphia chromosome is the hallmark of this disease [1]. CML are rare in childhren, constitutes around $3 \%$ of

Manuscript received: $27^{\text {th }}$ Feb 2016

Reviewed: $09^{\text {th }}$ March 2016

Author Corrected: $20^{\text {th }}$ March 2016

Accepted for Publication: $31^{\text {st }}$ March 2016 leukemias in children [2,3]. Usual initial presentation is chronic phase [4]. Blastic phase (BC) as initial presentation of CML accounts for only $10 \%$ of all cases [5]. We studied laboratory features including the clinical features of blastic phase of CML in children. PS, BMA, Cytogenetics, flow cytometry Immunophenotype (FCI) were studied in detail with the special note on molecular methods, including BCR-ABL transcripts and mutation analysis. Present study is the first exclusive and largest 
exclusive study on crisis phase of chronic myeloid leukemia in childhood from a single institution

\section{Methods}

We reviewed the records of 187 children (1-17 years), who were clinically suspected as CML in our institute between January 2004 to December 2014. Peripheral smear suggested CML in 101children. Bone marrow aspiration, cytogenetics, PCR and flow cytometry confirmed the diagnosis. Clinical features were recorded. Anaemia was diagnosed when the hemoglobin levels was $<10 \mathrm{~g} / \mathrm{dL}$. Leucocyte count $>100 \times 10^{9} / \mathrm{L}$ was seen in all cases. Thrombocytopenia $<150 \times 10^{9}$ was recorded.

\section{Results}

Most of the patients were $>10$ years of age ( 16 children). 7 children were between $<10$ years at initial diagnosis of CML. There was a male preponderance (13 boys, 9 girls) with a gender ratio of $1.4: 1$.

Abdominal fullness and pain was the most common symptom. Bleeding was seen in 2 patients. Only 2 patients had signs of leukostasis, such as head ache, dizziness, visual and hearing disturbances. Single patient had priapism as the primary complaint, he also had marked splenomegaly, Leucocyte count $>100 \times 10^{9} / \mathrm{L}$ was seen , and platelet count was low, LDH was $1607 \mathrm{U} / \mathrm{L}$.

Splenomegaly was the predominant finding occurring 95\% patients. Median size measured on ultrasound (USG) was 16 $\mathrm{cm}$. Mild to moderate hepatomegaly on USG was seen in most patients. 4 children had lymph node enlargement. One case with lymph node enlargement had priapism.

Blood tests for HIV, HBsAg and HCV were negative. Serum LDH levels range normal 45U/L to as high as 8980U/L. Serum Alkaline phosphatase was within normal range in all cases. Hemoglobin value ranged from (6.5-10.6g/dL) median hemoglobin was $5 \mathrm{~g} / \mathrm{dl}$. Anaemia was seen in $80 \%$ cases. WBC count $>100 \times 10^{9} / \mathrm{L}$ was seen in all cases. Platelet count ranged from $\left(35,000-11.26 \times 10^{9} / \mathrm{L}\right), 20$ cases had thrombocytopenia.

CML was Classified according to WHO 2008 criterias, Bone marrow analysis revealed blastic phase (fig1) in 22 cases( $20 \%$ ). Mean basophil was $8 \%$. Bone marrow basophilia $>6 \%$ were seen in all cases. Blast varied from $20 \%$ to $60 \%$.

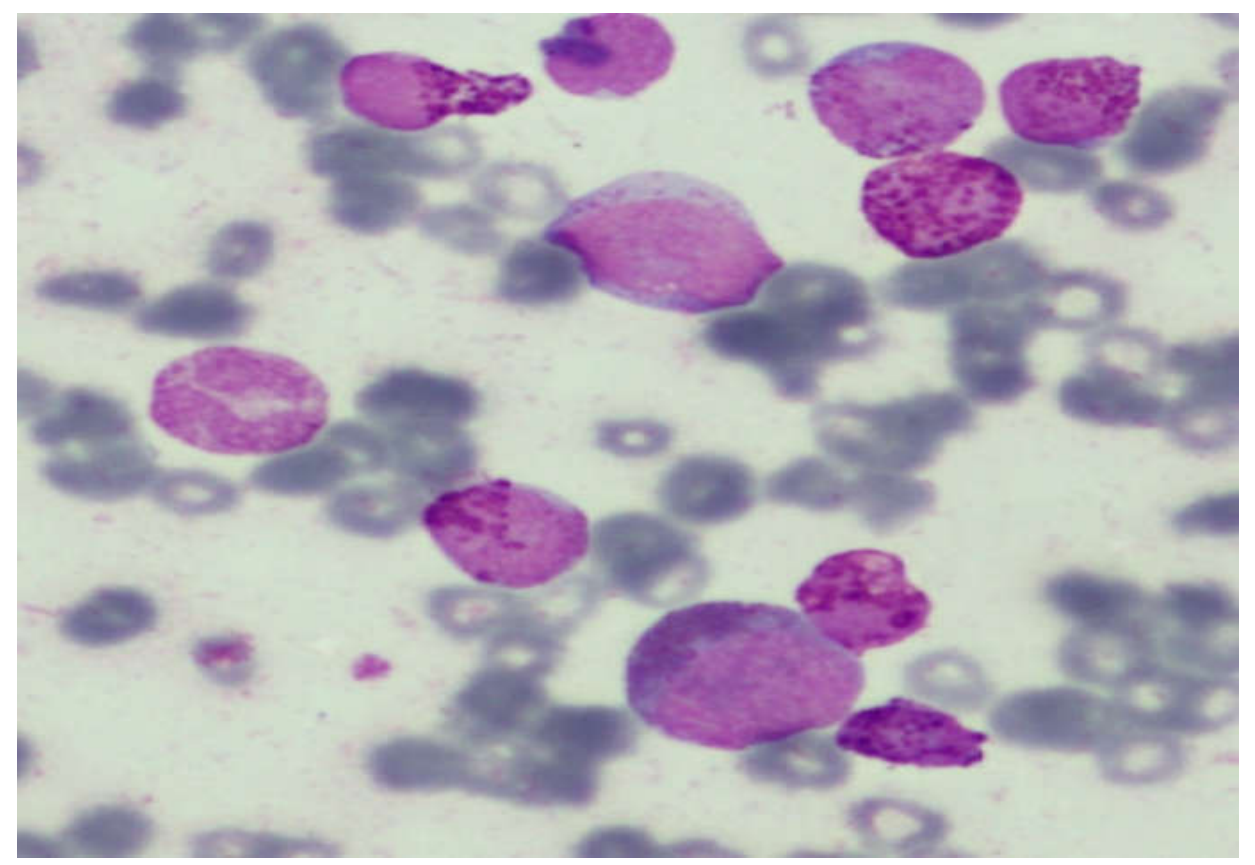

Fig-bone marrow aspiration showing increased blasts \&basophils. 

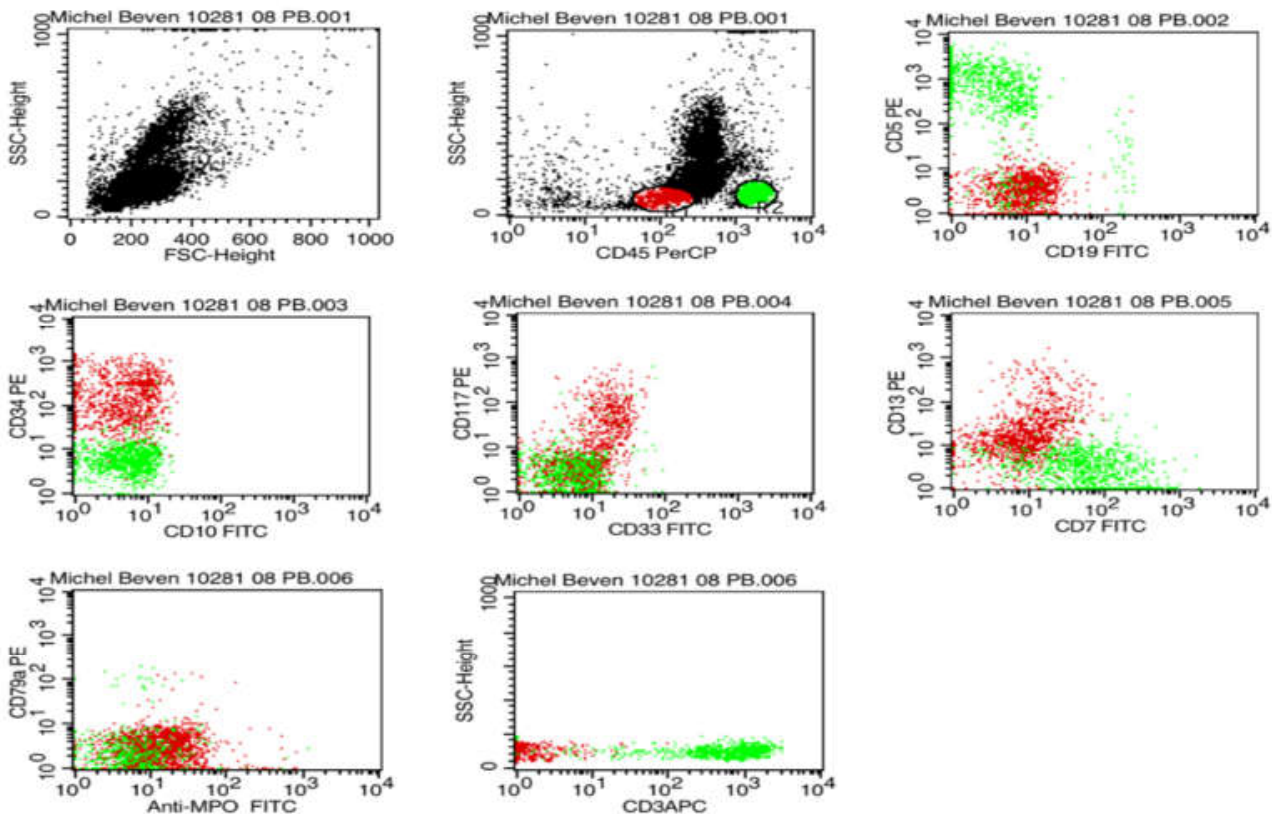

Gated cells in the blast region are

\# positive for CD $34, C D 33, C D 117, C_{13}$.

\# Negative for CD19, cCD3, CD5, CD7, CD10. cCD79a, MPO.

fig 2- flow scatterograms showing Myeloid blastic phase
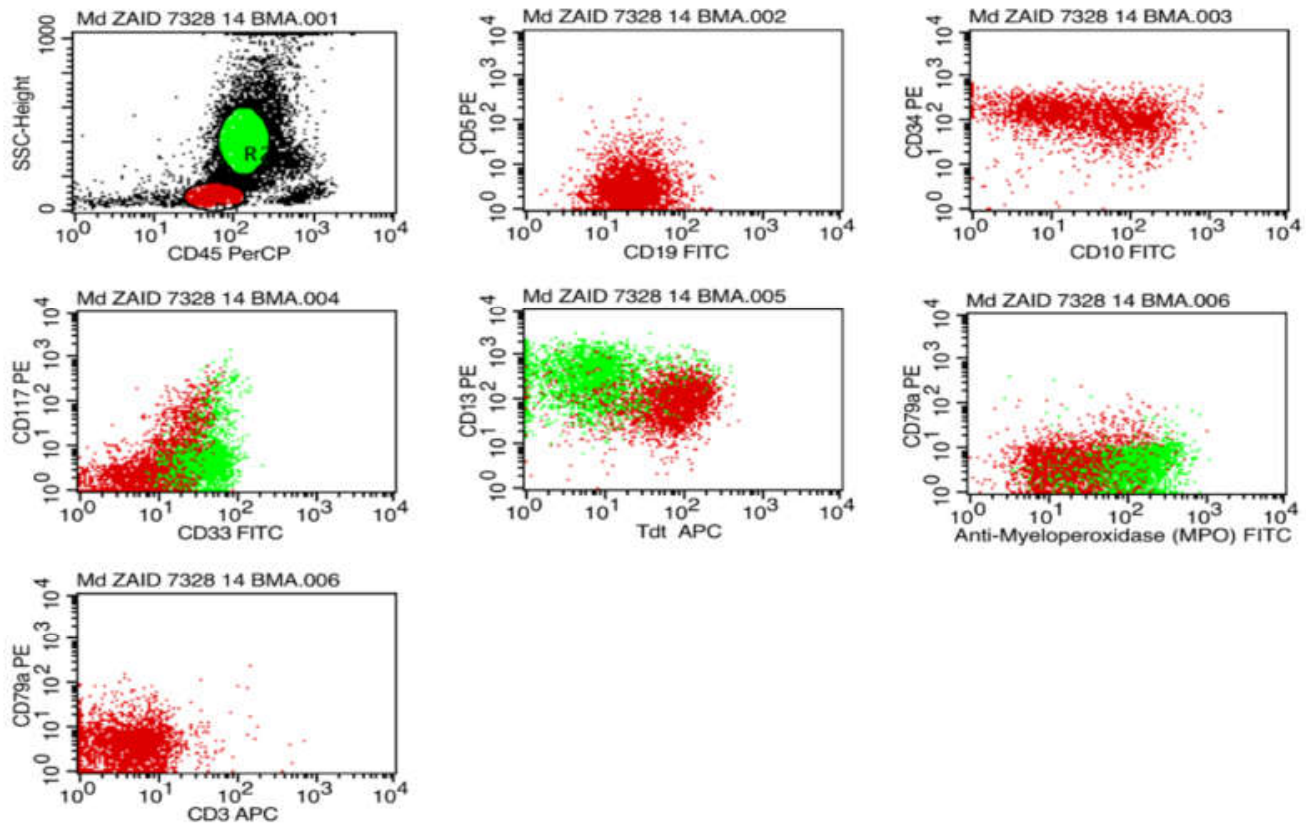

Gated cells in the blast region are

\# positive for: Tdt, CD34, CD19, CD10 with aberrant CD13.

\# Negative for: cCD79a,cCD3, CD5, CD33, CD117, MPO.

\section{fig 3-flow scatterograms showing lymphoid blastic phase}

The Phildelphia chromosome the hallmark cytogenetic abnormality of CML was identified in all 22 cases. 5 cases had additional chromosome abnormalities. Case 1: had 3 way translocation $\mathrm{t}(2.9: 22) \mathrm{p} 22$, q34, q11.case 2:myeloid blast crisis 
had der (9), t(9:22), q34:q11.2.i(17)q(10). flow in this case expressed CD13, CD33, CD117 however MPO was negative(fig2),e14qa2 transcript was evident on PCR. Case 3:Lymphoid blast crisis, had del8, del13, del14, del17, del19, add12,+3 mar, On flow cytometry Expression of CD 34, CD19, cCD79a, CD10 was seen(fig3). These blasts were negative for cCD3, CD5, CD7, MPO, CD33, CD13, CD117. Case4:had del(6q),Case5:had+11. FIC categorized the 8 cases as 5 myeloid and single mixed phenotype, 2 cases as lymphoid blastic phase.

\section{Discussion}

CML is called as disease of "firsts" due to many historical aspects $[6,7,8]$. CML results from the reciprocal translocation of genes on chromosome 9 and 22. This leads to juxta position of the BCR gene on chromosome 22 with the virus ABL gene. The fused BCR-ABL protein has altered tyrosine kinase activity $[9,10,11]$. It activates a number of intracellular signal transduction pathways, resulting in increasing myeloid proliferation and differentiation and decreasing apoptosis resulting clinically as CML. CML has become a paradigm of our understanding leukomogenesis $[6,7,1]$.

CML are extremely rare in childhood, constitutes around $3 \%$ of leukemias in children $[2,12,13]$. Its rarity was established by the following facts. Study from the children's oncology group included 23 centers \& 31 patients . A comparison between imatinib and stem cell transplant (SCT), as a treatment for childhood CML, included 18 patients in the SCT arm \& 30 patients in the IMA arm.

In other studies, the patient number varied from 4 to 39 [6]. These studies confirm the rarity of this disease in children. However higher incidence in Mumbai pediatric age group SEER registries was recorded $[11,14]$

In present study no association with radiation, therapeutic radiation, industrial chemicals, or alkylating agents [3] were noted confirming, no association of above said in children with CML. Natural history of CML is divided into chronic, accelerated and blast phase [3].

Most patients are diagnosed in chronic phase [1]. In the absence of treatment majority of patients progress from CP to BP either suddenly or through a transition AP. The transformed Phase are generally accompanied by worsened performance status and by symptoms related to severe anaemia, thrombocytopenia or marked splenic enlargement [1].

The data on clinical presentation of CML in children is sparse. This is the first reported series of CML in children which exclusively studied blastic phase of CML, incidentally this is the largest reported series till date. Also our study is the largest study from a single center, catering to patients from the lower socioeconomic status. In an analysis from the French

group, the maximum (47\%) number of patients belonged to the $10-14$ year age group Similar to the earlier study we had maximum number of patients between 10-15years age group that is 10 cases ( $45 \%$ ) . As found in the analysis from the French group \& millot [12], males predominated in numbers in our study also. This sex ratio may reflect a gender bias because of male preference for the access to treatment of this chronic disorder, more so in developing countries [13,14].

Asthenia and splenic discomfort were the predominant symptoms and splenomegaly was the predominant sign in our study. Thrombotic and haemorrhagic complication was not seen in the present study. Surprisingly we had large number (20\%)of the children presenting in progressive phase 22 cases ( CML-BP), this could probably reflect the health situation in developing countries where routine blood tests were not practiced as in developed countries and patients present in the late phase of disease.

Frederic Millot [15] et al, reported the presenting leukocyte counts to be higher in children, similar to his study,20children in present study presented with WBC count $>100 \times 10^{9} / \mathrm{L}$, however the exact increased WBC count couldnot be ascertained by the machine accurately in our institute. 2 cases presented in the blastic phase despite normal platelet count. 20 cases had thrombocytopenia.

Cytogenetic confirmation were available in all 22 cases and additional markers in 5 cases. This highlights importance of cytogenetics, where in cytogenetic analysis can monitor for the acquisition of clonal abnormalities and has an important role . BC as initial presentation of CML accounts for $10 \%$ of all cases [5], however study by Hugo Castro et al,Blast crisis was observed in $78 \%$ of cases ${ }^{[16]}$. 
In the present study blastic phase of CML(20\%). In the BP the blast lineage may be obvious morphologically but often the blast are primitive or heterogenous and cytochemical and immunophenotypic analysis is recommended [1] . 60-80\% BP are myeloid [1], Similar to earlier studies we had $62 \%$ of $\mathrm{BP}$ in myeloid crisis. In Myeloid BP the blasts may have strong, weak or no myeloperoxidase activity, but express antigen associated with granulocytic, monocytic, megakaryoblastic or erythroid differentiation $[1,17]$.

$20 \%$ of BP are Lymphoid. Most cases of Lymphoblastic $\mathrm{BP}$ are precursor B in origin $[1,17]$, but $\mathrm{T}$ lymphoblastic origin also occur $[1,17,18,19]$. $25 \%$ of cases were in lymphoid BP in our study, and all of them belonged to Blineage. $25 \%$ of BP fulfills criteria for mixed lineage [1]. We had a single case of mixed lineage.

The discovery of Imatinibmesylate completely changed standard therapeutic approaches for all phases of CML. The promise of targeted therapy for CML was approved for the first time in 2001.Imatinib received accelerated approval by AUS FDA in 2003 for the use in pediatric CML [6], however there are very few literature available regarding Imatinib use in children. Imatinib occupies the adenosine triphosphate binding site in the $\mathrm{SH} 1$ domain of the BCR-ABLoncoprotein [3].

Similar to many earlier studies [20], Imatinibmesylate was used as first line therapy in patients with chronic myeloid leukemia in blastic phase. Imatinib was generally well tolerated as in earlier studies [21]. All Imatinib treated patients attained remission, and are on survived for mean of 6 months, continue to be on follow up and are in remission. Patients in accelerated or blast crisis or who fail to respond or responds suboptimally for TKIs either because of intolerance or resistance, should pursue stem cell transplantation [22].

It is important to follow up the patient to know the cause of rising BCR-ABL levels by both mutation and cytogenetic analysis. Detection of $2^{\text {nd }} \mathrm{Ph}$ chromosome indicates amplification of the BCR-ABL gene, as a mechanism of Imatinib resistance [9].

In our study 7 cases underwent kinase domain mutation analysis. However no mutations were detected in these 7 cases. Recommendation for patients with such mutations is stem cell transplant, different kinase inhibitor, or investigational treatments. Estimated 12 month overall survival in advanced phase of CML was $75 \%$ [12].
All the Imatinib treated patients achieved remission and are alive at median age of 6 months and continue to be on follow up. This study represents the largest ,first exclusive series of blastic phase of CML in children in which the clinical signs, biology and laboratory parameters including PS, BMA, Cytogenetics, PCR and FIC were studied in detail. In the era of successful BCR-ABL kinase inhibitor treatment for CML patients, molecular monitoring, mutation and cytogenetic analysis helps to know the cause of imatinib resistance [9].

There is definite need for more dialogue between the medical fraternity in the developing and more affluent countries especially regarding the so called rare diseases in affluent countries, which is not so rare in developing countries like India. There is need to create and fund more venues for such dialogue. We have a moral responsibility towards all $\mathrm{cml}$ children to provide curative therapies so that each life can be lived to its fullest.

Abbreviations: CML: chronic myeloid leukemia.WBC: white blood cell. SCT: stem cell transplant. PCR: polymerase chain reaction.PS: peripheral smear. BMA: bone marrow aspiration, BP:blastic phase

Funding: Nil, Conflict of interest: None. Permission of IRB: Yes

\section{References}

1. J W Vardiman,J.V.Melo,M.Baccarani,J.Thiele. Chronic Myeloid Leukemia,BCR-ABL1 positive. WHO classification of hematopoietic and lymphoid tissue $4^{\text {th }}$ ed.IARC,Lyon,France:2008.pp 32-37

2. Alkhatib MA. Pattern of pediatric chronic myeloid leukemia in Sudan and hematological response to imatinib. Pediatr Hematol Oncol. 2011 Mar;28(2):1005. doi: 10.3109/08880018.2010.512949. Epub 2010 Nov 17.

3. Philip A Pizzo,David G Poplock.Principles and practice of pediatric oncology .Arnold J Altman.Cecilia FN. Chronic leukemias of childhood $6^{\mathrm{TH}}$ edition .Lippincott Williams and wilkins.2011:611-637

4. K Ganessan , RuchikaGoel,Kamleshkumar,SameerBakshi.Biphenotyp ic extramedullary blast crisis as a presenting manifestation of Phildelphia chromosome-Positive 
CML in a child.Pediatric Hematology and Oncology .2007;24:195-198.

5. LarsonRS, Wolff SN.Chronic Myeloid Leukemia. In: LeeGR, forester J,LukensJ, paraskeras F,GreerJP, Rodgers GM,eds .Wintrobe's clinical hematology, $10^{\text {th }}$ ed.Baltimore:Williams\&Wilkin's;1999: 2342-2373.

6. Raut L, Bohara VV, Ray SS, Chakrabarti P, Chaudhuri U. Chronic myeloid leukemia in children and adolescents: A single center experience from Eastern India. South Asian J Cancer. 2013 Oct;2(4):260-4. doi: 10.4103/2278-330X.119891.

7. LalitRaut .Chronic myeloid leukemia in childrenA brief review.Clin Cancer Investigation journal.2014;3:467-471.

8. Stefan Faderl,ZeevEstrovRazelleKurzrock , hagop M $\mathrm{K}$ antarjian .The biology of Chronic Myeloid Leukemia. The New England Journal of Medicine.1999;15;165-172.

9. ThomasErnsrt and Andreas Hochhaus . Chronic Myeloid Leukemia:Clinical impact of BCR-ABL1 Mutations and other lesions Associated With Disease Progression. Seminars in Oncology.2012;39:58-66.

10. Goldman JM, Melo JV. Chronic myeloid leukemia-advances in biology and new approaches to treatment. N Engl J Med. 2003 Oct 9;349(15):1451-64.

11. Dikshit RP, Nagrani R, Yeole B, Koyande S, Banawali S. Changing trends of chronic myeloid leukemia in greater Mumbai, India over a period of 30 years. indian J Med Paediatr Oncol. 2011 Apr;32(2):96100. doi: 10.4103/0971-5851.89792.

12.Millot F, Traore P, Guilhot J, Nelken B, Leblanc T, Leverger G, Plantaz D, Bertrand Y, Bordigoni P, Guilhot F. Clinical and biological features at diagnosis in 40 children with chronic myeloid leukemia. Pediatrics. 2005 Jul;116(1):140-3

13. Ching-Hon Pui ,Childhood Leukemias. The New England Journal Of Medicine.1995;332:1620-1628

14. Wechalekar AD, Parande CM. Haematological malignancies in developing countries: is CML the commonest childhood leukaemia? Leukemia. 2007 Oct;21(10):2194; author reply 2195. Epub 2007 Aug 2.

15. Millot $F$

,GuilhotJ,NelkenB,LeblancT,DeBontES,BekassyAN,et al Imatinibmesylate is effective in children with Chronic Myelogenous Leukemia in late chronic and advanced phase and in relapse after stem cell transplantation.Leukemia.2006;20:187-92.

16. Castro-Malaspina H, Schaison G, Briere J, Passe S, Briere J, Pasquier A, Tanzer J, Jacquillat C, Bernard J. Philadelphia chromosome-positive chronic myelocytic leukemia in children. Survival and prognostic factors. Cancer. 1983 Aug 15;52(4):721-7.

17. Khalidi HS, Brynes RK, Medeiros LJ, Chang KL, Slovak ML, Snyder DS, Arber DA. The immunophenotype of blast transformation of chronic myelogenous leukemia: a high frequency of mixed lineage phenotype in "lymphoid" blasts and A comparison of morphologic, immunophenotypic, and molecular findings. Mod Pathol. 1998 Dec;11(12):1211-21.

18. Voican I, Vlădăreanu AM, Bumbea H, Begu M. Sudden blast crisis in a chronic myeloid leukemia patient during imatinib therapy. Rom J Intern Med. 2012 Jul-Sep;50(3):241-4.

19. Kim AS, Goldstein SC, Luger S, Van Deerlin VM, Bagg A. Sudden extramedullary T-lymphoblastic blast crisis in chronic myelogenous leukemia: a nonrandom event associated with imatinib? Am J Clin Pathol. 2008 Apr;129(4):639-48. doi: 10.1309/GTGTEQAFMV30W753.

20. Thota NK, Gundeti S, Linga VG, Coca P, Tara RP; Raghunadharao. Imatinib mesylate as first-line therapy in patients with chronic myeloid leukemia in accelerated phase and blast phase: a retrospective analysis. Indian J Cancer. 2014 Jan-Mar;51(1):5-9. doi: 10.4103/0019-509X.134598.

21. Belgaumi AF, Al-Shehri A, Ayas M, Al-Mahr M, Al-Seraihy A, Al-Ahmari A, El-Solh H. Clinical characteristics and treatment outcome of pediatric patients with chronic myeloid leukemia. Haematologica. 2010 Jul;95(7):1211-5. doi: 10.3324/haematol.2009.015180. Epub 2010 Apr 21.

22. Andolina JR, Neudorf SM, Corey SJ. How I treat childhood CML. Blood. 2012 Feb 23;119(8):1821-30. doi: 10.1182/blood-2011-10-380774. Epub 2011 Dec 30 . 
How to cite this article?

Namrata N Rajkumar, Raghvendra H Vijay, Vijay C Raghu, Lakshmiah K C. Flow immunophenotyping features of crisis phase of chronic myeloid leukemia in childhood: do we really care?: Int J Med Res Rev 2016;4(3):463-469. doi: 10.17511/ijmrr.2016.i03.31. 